\title{
OsteoLuban a Novel Natural Remedy for Osteoarthritis
}

\author{
Luay Rashan ${ }^{1}$ and Rafie Hamidpour*1,2 \\ ${ }^{1}$ Biodiversity Center, University of Dhofar, Oman \\ ${ }^{2}$ Department of Herbal Medicine, Pars Bioscience Research Centerm, United States
}

*Corresponding author: Rafie Hamidpour, Pars Bioscience Research Center USA 14109 Cambridge Lane Leawood, Kansas 66224, United States.

To Cite This Article: Rafie Hamidpour. OsteoLuban a Novel Natural Remedy for Osteoarthritis. Am J Biomed Sci \& Res. 2019 - 5(6). AJBSR. MS.ID.000980. DOI: 10.34297/AJBSR.2019.05.000980.

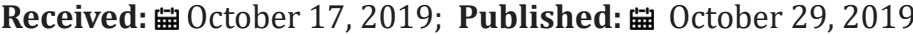

\begin{abstract}
Osteoarthritis (OA) is the most common type of arthritis. It is also known as degenerative arthritis or degenerative joint disease or "wear and tear" arthritis. It occurs when the cartilage or cushion between joints breaks down leading to pain, stiffness and swelling. The most common symptoms of osteoarthritis are stiffness, particularly first thing in the morning or after resting, and pain. Affected joints may get swollen after extended activity. $\mathrm{OA}$ is the major cause of disability in both the developed and developing countries. The worldwide estimate for symptomatic OA is $9.6 \%$ among men and $18 \%$ among women. In USA only OA affects approximately 27 million people. Osteoarthritis has no specific cause, however, there are several factors lead to the development of OA including excess weight, injury or overuse and genes, among others. As far as treatment of OA is concerned, this disease cannot be reversed, but treatment can reduce primary pain. There are several options that can help reduce pain and helps patients move better. It includes using oral non-steroidal anti-inflammatory drugs (NSAIDs such as aspirin, ibuprofen, Nabumetone and naproxen.

It is sometimes possible to use NSAIDs temporarily and then discontinue them for periods of time without recurrent symptoms, thereby decreasing the risk of side effects. Further, topical diclofenac topical gel (voltaren) is used to relieve osteoarthritis pain in the knees. It works by stopping the body's production of a substance that causes pain. Among other conventional treatment of OA is surgery, joint injection of glucocorticoids and hyaluronic acid. On the other hand, there are several non-conventional treatments used for treating OA including glucosamine and chondroitin sulfate, curcumin, Boswellia serrata extract and others. In addition, acupuncture, electrostimulation and electromagnetic field and others are also used. We describe in this paper a new oral herbal remedy oral that consists of three monographic herbs with potent anti -inflammatory and analgesic properties that make it a promising treatment for osteoarthritis.
\end{abstract}

\section{Introduction}

Osteoarthritis $(\mathrm{OA})$ is a type of joint disease that results from breakdown of joint cartilage and underlying bone [1]. OA is sometimes called degenerative joint disease or degenerative arthritis. $\mathrm{OA}$ is the most common chronic condition of the joints, progressive and debilitating. It can affect any joint, but it occurs most often in knees, hips, lower back and neck, small joints of the fingers and the bases of the thumb and big toe. It affects millions of people worldwide [2]. In normal joints, cartilage covers and protects the ending part of the bones, promoting friction and absorbing impacts. The progressive joint destruction leads to lameness, pain, mobility limitations and comprises the overall quality of life [2]. The most commonly involved joints are those near the ends of the fingers, at the base of the thumb, neck, lower back, knee, and hips [3]. Joints on one side of the body are often more affected than those on the other [2]. Causes of OA include previous joint injury, abnormal joint or limb development, and inherited factors $[3,4]$. Risk is greater in those who are overweight, have legs of different lengths, or have jobs that result in high levels of joint stress [3-5]. OA is believed to be caused by mechanical stress on the joint and low-grade inflammatory processes [7]. It develops as cartilage is lost and the underlying bone becomes affected [3] as pain may make it difficult to exercise, muscle loss may occur $[3,6]$. Worldwide estimation reported over 100 million people globally suffer from $\mathrm{OA}$, which is one of the most common causes of disability $[6,7]$. As per the WHO report on disability (2011), the prevalence of moderate and severe disability (in millions) due to OA in high-income countries was 1.9 and 8.1 in the age group of 0-59 and above 60 years, respectively. In the low- and middle-income countries, these figures were 14.1 and 19.4 [8]. The worldwide prevalence estimate for symptomatic OA is $9.6 \%$ among men and almost double (18\%) among women [9].

Among the factors that can increase your risk of osteoarthritis include: 
1. Older age. The risk of osteoarthritis increases with age.

2. Sex. Women are more likely to develop osteoarthritis, though it isn't clear why.

3. Obesity. Carrying extra body weight contributes to osteoarthritis in several ways, and the more you weigh, the greater your risk. Increased weight adds stress to weightbearing joints, such as your hips and knees. Also, fat tissue produces proteins that can cause harmful inflammation in and around your joints.

4. Joint injuries. Injuries, such as those that occur when playing sports or from an accident, can increase the risk of osteoarthritis. Even injuries that occurred many years ago and seemingly healed can increase your risk of osteoarthritis.

5. Repeated stress on the joint. If your job or a sport you play places repetitive stress on a joint, that joint might eventually develop osteoarthritis.

6. Genetics. Some people inherit a tendency to develop osteoarthritis.

7. Bone deformities. Some people are born with malformed joints or defective cartilage.

8. Certain metabolic diseases. These include diabetes and a condition in which your body has too much iron (hemochromatosis).

Diagnosis is made with reasonable certainty based on history and clinical examination $[10,11]$. X-rays may confirm the diagnosis. The typical changes seen on X-ray include: joint space narrowing, subchondral sclerosis (increased bone formation around the joint), subchondral cyst formation, and osteophytes [12]. Usually other imaging techniques are not necessary to clinically diagnose osteoarthritis. In 1990, the American College of Rheumatology, using data from a multi-center study, developed a set of criteria for the diagnosis of hand osteoarthritis based on hard tissue enlargement and swelling of certain joints [13]. These criteria were found to be $92 \%$ sensitive and $98 \%$ specific for hand osteoarthritis versus other entities such as rheumatoid arthritis and spondyloarthropathies [13]. On the other hand, analyzing blood or joint fluid can help confirm the diagnosis. Concerning treatment of $\mathrm{OA}$, there are several options that can be categorized as follows:

\section{Conventional Medicine}

\section{By mouth}

The first line of treatment for $\mathrm{OA}$ by mouth is pain medication which is a NSAIDs drug represented by paracetamol (acetaminophen) [14,15]. Pain relief does not differ according to dosage [16]. For mild to moderate symptoms effectiveness of acetaminophen NSAIDs such as naproxen, though for more severe symptoms NSAIDs may be more effective [14]. NSAIDs are associated with greater side effects such as gastrointestinal bleeding [14]. Another class of NSAIDs, COX-2 selective inhibitors (such as celecoxib) are equally effective when compared to nonselective NSAIDs [17]. Opioids by mouth, including both weak opioids such as tramadol and stronger opioids, are also often prescribed. Oral steroids are not recommended in the treatment of osteoarthritis [15].

\section{Topical}

There are several NSAIDs available for topical use, including diclofenac the use of topical capsaicin to treat osteoarthritis is controversial, as some reviews found benefit $[18,19]$ while others did not [20].

\section{Joint injections}

Joint injection of glucocorticoids (such as hydrocortisone) leads to short term pain relief that may last between a few weeks and a few months [21]. Injections of hyaluronic acid have not produced improvement compared to placebo for knee arthritis [22,23].

\section{Surgery}

Includes joint replacement surgery or resurfacing.

\section{Complimentary medicine}

\section{Glucosamine and chondroitin}

The effectiveness of glucosamine is controversial [24]. Reviews have found it to be equal to $[25,26]$ or slightly better than placebo $[27,28]$. A 2015 Cochrane review of clinical trials of chondroitin found that most were of low quality, but that there was some evidence of short-term improvement in pain and few side effects; it does not appear to improve or maintain the health of affected joints [29].

\section{other remedies}

Avocado-soybean unsaponifiables (ASU) is an extract made from avocado oil and soybean oil [30] that is sold under many brand names worldwide as a dietary supplement [31] and as a drug in France. A few high-quality studies of Boswellia serrata show consistent, but small, improvements in pain and function [30]. Curcumin [32], phytodolor [18], and s-adenosyl methionine (SAMe) [18], may be effective in improving pain.

On the other hand, there is little evidence supporting benefits for some supplements, including:

1. The Ayurvedic herbal preparations with brand names Articulin F and Eazmov

2. Duhuo Jisheng Wan, a Chinese herbal preparation

3. Fish liver oil

4. Ginger

5. Russian olive; the herbal preparation gitadyl 
6. Omega-3 fatty acids

7. The brand-name product Reumalax

8. Stinging nettle

9. Vitamins $\mathrm{A}, \mathrm{C}$, and $\mathrm{E}$ in combination

10. Vitamin E alone

11. Vitamin $\mathrm{K}$

12. Vitamin D

13. Collagen

14. Willow bark

There is insufficient evidence to make a recommendation about the safety and efficacy of these treatments $[18,33]$.

\section{Acupuncture and other interventions}

While acupuncture leads to improvements in pain relief, this improvement is small and may be of questionable importance [34]. Waiting list-controlled trials for peripheral joint osteoarthritis do show clinically relevant benefits, but these may be due to placebo effects $[35,36]$. Acupuncture does not seem to produce long-term benefits. Electrostimulation techniques such as TENS have been used for twenty years to treat osteoarthritis in the knee, however there is no conclusive evidence to show that it reduces pain or disability. Further research is needed to determine if balnotherapy for osteoarthritis (mineral baths or spa treatments) improves a person's quality of life or ability to function [37]. The use of ice or cold packs may be beneficial; however, further research is needed. OsteoLuban (Figure2) is a new class of herbal- based evidence remedy that contains monographic herbal ingredients which is particularly designed for those suffering from OA. OsteoLunab exhibited potential anti- inflammatory and analgesic properties both in vitro and in vivo and also in clinical studies. This OTC product is superior in its action compared to some non-steroidal anti- inflammatory and analgesic drugs used currently in the clinics such as Aspirin, Brufen and paracetamol as shown in our in vivo and clinical studies. The pharmacological action reported by this product indicated its action on the inflammatory response by blocking release of proinflammatory cytokines and other signaling pathways. Therefore, it is a class of products that can be used for $\mathrm{OA}$ and other related diseases such as rheumatism. This product is not only pain relief but also has potential effect in reducing "inflammation".

\section{Description}

OsteoLuban is a very distinguished product that developed after careful preclinical and clinical studies. Several in vitro and in vivo studies were conducted to study the efficacy and safety of this product both in vitro and in vivo. For in vitro studies, several human and murine cell lines were used including primary murine microglia, raw mouse macrophages, primary human monocytes and primary human fibroblasts to see its effect on prostaglandin E2, interleukin 1-beta (IL-1 Beta), tumor necrosis factor (TNF) and interleukin6 (IL6). These studies showed that the product possess significant anti - inflammatory properties.

For in vivo studies, the activity of the product was studied in albino rats using two different pharmacological screening tests, these are:

1. Inhibition of ascites using albino rats

2. Freund's adjuvant using albino rats

OsteoLuban exhibited a potential anti-inflammatory activity compared to phenylbutazone drug in causing a diminution of ascites fluid. Further, another confirmatory result from Freund's adjuvant test which clearly showed that OsteoLuban is more active compared with the standard drugs used in this test represented by brufen and aspirin.

Further, the analgesic property of OsteoLuban was evaluated using two pharmacological screening tests, these are:

1. Writhing induced by chemicals using albino mice

2. Hot plate test using albino mice

OsteoLuban was more potent as analgesic in both tests compared with the reference standard used in the two above tests represented by paracetamol. On the other hand, the oral acute toxicity of OsteoLuban was investigated in vivo utilizing healthy experimental mice as a model. A single dose was administered to the animals followed by monitoring for a period of 14 days after dosing and recording death and changes in animal behavior and any other physical variables. The results obtained indicated that the oral LD50 of OsteoLuban is at least greater than $2000 \mathrm{mg} / \mathrm{kg}$ in balb/c mice. In addition, OsteoLuban neither induced any death nor caused any abnormal behavior when tested at a dose of $2000 \mathrm{mg} /$ $\mathrm{kg}$.

\section{Properties of OsteoLuban}

1. Helps in easing pain and reducing inflammation

2. Helps in maintaining healthy joints, cartilages and tendons

3. Helps to treat osteoarthritis and rheumatoid arthritis

4. Helps for short term relief of low back pain

5. Helps for the relief of minor joint and articular pain

6. Has antioxidant properties

\section{Healing Paradigm}

The healing paradigm of OsteoLuban as shown below (Figure1\&2), is that its uses encourage a person to maintain a healthy joint by using natural supplement. 

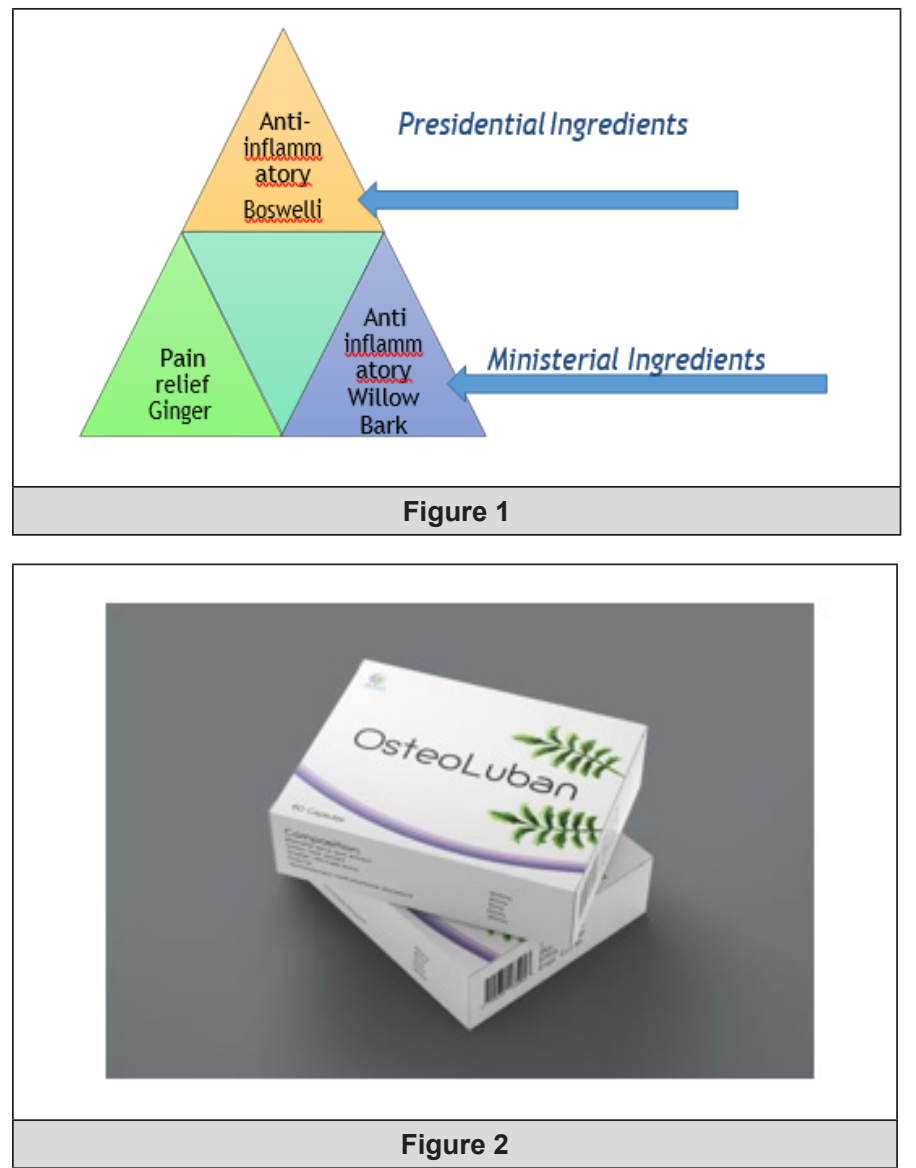

\section{Result}

OsteoLuban is a product which consist of selected and unique blend of three monographic and well- researched herbs (Boswellia sacra, Salix alba and Zingiber offininale extracts) and with diversified actions since this product includes six boswellic and lupeolic acids, polyphenols, flavonoids, salicin, terpenes and other compounds. This unique combination of ingredients potentiates their effect on the body. According to feedback received from clinicians who tested this product on patients suffering from osteoarthritis, the inflammation in patients with $\mathrm{OA}$ is reduced by blocking pro- inflammatory 5-lipoxygenease (5-LOX). This enzyme is the first enzyme in the metabolic pathway leading to the synthesis of leukotrienes, which are harmful substances that scientists believe may have direct influence on a number of diseases process. Further, with reduced "inflammation", nerve irritation will also decease which lead to reduced pain sensation. Being an antioxidant, OsteoLuban facilitates removal of free radicals in the body that causes damage to the body structures including joints, cartilages, tendons, and synovial membrane. Finally, it seems that the medicinal ingredients in OsteoLuban capsule work in an effective and synergistic way to support each claims, which on the other hand, support the healing paradigm.

\section{Properties of OsteoLuban}

OsteoLuban is a unique natural health product composed of an extract obtained from Boswellia sacra gum resin using special extraction procedure [38] and was characterized using HPLC/ MS/MS [39]. The pharmacological action of this extract showed potential anti-inflammatory and analgesic effects when compared with standard drugs used in clinics. Further, it contains willow bark with salicin which hydrolyzed in the gastrointestinal tract to give salicyl alcohol and D-glucose which upon absorption, salicyl alcohol oxidized into salicylic acid (Aspirin) and other compounds. Finally, it contains ginger which helps in pain relief from both osteoarthritis and rheumatoid arthritis, migraine headache and prevent nausea. Therefore, the medicinal ingredients in OsteoLuban work in an effective and synergistic way to support each of the claims, which on the other hand, support the healing paradigm.

\section{Side effects}

No serious side effects were reported from using this product. Some more than $25 \%$ patients using this product experienced minor to moderate stomach pain.

\section{Contraindications}

OsteoLuban is contraindicated in patients with stomach ulcers, kidney disease and hypothrombinemia. Also, it is contraindicated to patient sensitive to aspirin and willow bark. Not to be used during pregnancy.

\section{Direction for use}

Three capsules daily in three dividing doses with meals.

\section{References}

1. Arden N, Blanco F, Cooper C, Guermazi A, Hayashi D, et al. (2015) Atlas of Osteoarthritis. Springer Pp. 21.

2. Martello E, Bigliati M, Bisanzio D, Biasibetti E, Dosio F, et al. (2019) Effects on pain mobility of a new diet supplement in dogs with osteoarthritis: A pilot Study. Annals of Clinical Laboratory Research 7(2): 304.

3. (2015) "Osteoarthritis". National Institute of Arthritis and Musculoskeletal and Skin Diseases.

4. Glyn Jones S, Palmer AJ, Agricola R, Price AJ, Vincent TL, et al, (2015) Osteoarthritis. Lancet 386(9991): 376-387.

5. Vingård E, Englund M, Järvholm B, Svensson O, Stenström K, et al. (2016) Occupational Exposures and Osteoarthritis: A systematic review and assessment of medical, social and ethical aspects. SBU Assessments (Report) Stockholm: Swedish Agency for Health Technology Assessment and Assessment of Social Services (SBU) No. 253.

6. Conaghan P (2014) Osteoarthritis - Care and management in adults. National Clinical Guideline Centre (UK).

7. Di Puccio F, Mattei L (2015) Biotribology of artificial hip joints. World J Orthop 6(1): 77-94.

8. March L, Smith EU, Hoy DG, Cross MJ, Sanchez Riera L, et al. (2014) Burden of disability due to musculoskeletal (MSK) disorders. Best Pract Res Clin Rheumatol 28(3): 353-366.

9. Elsternwick (201 Zhang W, Doherty M, Peat G, Bierma Zeinstra MA Arden NK, Bresnihan B, Herrero Beaumont G, Kirschner S, Leeb BF, Lohmander LS, Mazières B, Pavelka K, Punzi L, So AK, Tuncer T, Watt I, Bijlsma JW (2010).

10. Zhang W, Doherty M, Peat G, Bierma Zeinstra MA, Arden NK, et al. (2010) EULAR evidence-based recommendations for the diagnosis of knee osteoarthritis. Ann Rheum Dis 69(3): 483-489. 
11. Bierma Zeinstra SM, Oster JD, Bernsen RM, Verhaar JA, Ginai AZ, et al. (2002) Joint space narrowing and relationship with symptoms and signs in adults consulting for hip pain in primary care. J Rheumatol 29(8): 1713-1718.

12. Kalunian KC (2013) Patient information: Osteoarthritis symptoms and diagnosis (Beyond the Basics). UpToDate.

13. Altman R, Alarcón G, Appelrouth D, Bloch D, Borenstein D, et al. (1990) The American College of Rheumatology criteria for the classification and reporting of osteoarthritis of the hand. Arthritis Rheum 33(11): 16011610.

14. Flood J (2010) The role of acetaminophen in the treatment of osteoarthritis. Am J Manag Care. 16: S48-S54.

15. Zhang W, Moskowitz RW, Nuki G, Abramson S, Altman RD, et al (2007) OARSI recommendations for the management of hip and knee osteoarthritis, part I: critical appraisal of existing treatment guidelines and systematic review of current research evidence. Osteoarthritis Cartilage. 15(9): 981-1000.

16. Flood J (2010) The role of acetaminophen in the treatment of osteoarthritis. Am J Manag Care 16: S48-S54.

17. Karabis A, Nikolakopoulos S, Pandhi S, Papadimitropoulou K, Nixon R et al. (2016) High correlation of VAS pain scores after 2 and 6 weeks of treatment with VAS pain scores at 12 weeks in randomised controlled trials in rheumatoid arthritis and osteoarthritis: meta-analysis and implications. Arthritis Res Ther 18: 73.

18. De Silva V, El Metwally A, Ernst E, Lewith G, Macfarlane GJ (2011) Evidence for the efficacy of complementary and alternative medicines in the management of osteoarthritis: a systematic review. Rheumatology (Oxford) 50(5): 911-920.

19. Cameron M, Gagnier JJ, Little CV, Parsons TJ, Blümle A, et al. (2009) Evidence of effectiveness of herbal medicinal products in the treatment of arthritis. Part I: Osteoarthritis. Phytother Res 23(11): 1497-1515.

20. Altman R, Barkin RL (2009) Topical therapy for osteoarthritis: clinical and pharmacologic perspectives. Postgrad Med 121(2): 139-147.

21. Arroll B, Goodyear Smith F (2004) Corticosteroid injections for osteoarthritis of the knee: meta-analysis. BMJ 328(7444): 869.

22. Rutjes AW, Jüni $P$, da Costa $B R$, Trelle $S$, Nüesch $E$, et al. (2012) Viscosupplementation for osteoarthritis of the knee: a systematic review and meta-analysis Ann Intern Med 157(3): 180-191.

23. Jevsevar D, Donnelly P, Brown GA, Cummins DS (2015) Viscosupplementation for Osteoarthritis of the Knee: A Systematic Review of the Evidence. J Bone Joint Surg Am 97(24): 2047-2060.

24. Burdett N, McNeil JD (2012) Difficulties with assessing the benefit of glucosamine sulphate as a treatment for osteoarthritis. Int J Evid Based Healthc 10(3): 222-226.

25. Wandel S, Jüni P, Tendal B, Nüesch E, Villiger PM, et al. (2010) Effects of glucosamine, chondroitin, or placebo in patients with osteoarthritis of hip or knee: network meta-analysis. BMJ 341: c4675.
26. Wu D, Huang Y, Gu Y, Fan W (2013) Efficacies of different preparations of glucosamine for the treatment of osteoarthritis: a meta-analysis of randomised, double-blind, placebo-controlled trials. Int J Clin Pract 67(6): 585-594.

27. Chou R, McDonagh MS, Nakamoto E, Griffin J (2011) Analgesics for Osteoarthritis: An Update of the 2006 Comparative Effectiveness Review. Comparative Effectiveness Reviews. 38. Agency for Healthcare Research and Quality (AHRQ).

28. Miller KL, Clegg DO (2011) Glucosamine and chondroitin sulfate. Rheumatic Diseases Clinics of North America 37(1): 103-18.

29. Singh JA, Noorbaloochi S, MacDonald R, Maxwell LJ (2015) Chondroitin for osteoarthritis. Cochrane Database Syst Rev p. 1.

30. Cameron M, Chrubasik S (2014) Oral herbal therapies for treating osteoarthritis. Cochrane Database Syst Rev 5: CD002947.

31. Christiansen BA, Bhatti S, Goudarzi R, Emami S (2015) Management of Osteoarthritis with Avocado/Soybean Unsaponifiables. Cartilage 6(1): 30-44.

32. Grover AK, Samson SE (2016) Benefits of antioxidant supplements for knee osteoarthritis: rationale and reality. Nutr J 15: 1.

33. Hussain S, Singh A, Akhtar M, Najmi AK(2017) Vitamin D supplementation for the management of knee osteoarthritis: a systematic review of randomized controlled trials. Rheumatol Int 37(9): 1489-1498.

34. Lin X, Manheimer E, Cheng K, Linde K, Lao L, et al. (2010) Acupuncture for peripheral joint osteoarthritis. Cochrane Database Syst Rev 1: CD001977.

35. Huang K, Zhu G, Huang Z, Qin A, Fan S (2016) The Effects of Acupuncture on Chronic Knee Pain Due to Osteoarthritis: A Meta-Analysis. J Bone Joint Surg Am 98(18): 1578-1585.

36. Manheimer E, Cheng K, Wieland LS, Shen X, Lao L, et al. (2018) Acupuncture for hip osteoarthritis. Cochrane Database Syst Rev 5: CD013010.

37. Verhagen AP, Bierma Zeinstra SM, Boers M, Cardoso JR, Lambeck J, et al. (2007) Balneotherapy for osteoarthritis. Cochrane Database Syst Rev 4: CD006864.

38. Rashan L, Hasson SSA, Simmet T, Mohammed SAA, Al Jabri AAH (2019) Boswellic Acid Extraction. South African Patent Application No. 2019/02740, Filing Date 2 May 2019.

39. Schmiech M, Lang SJ, Werner K, Rashan LJ, Syrovets T, et al. (2019) Comparative analysis of pentacyclic triterpenic acid composition of oleaogum resins of different Boswellia species and their in vitro cytotoxicity against treatment- resistant human breast cancer cells. Molecules 24(11). 